\title{
Videothoracoscopy in the treatment of mediastinal cysts
}

\author{
Daniel Brzeziński, Mariusz P. Łochowski, Józef Kozak \\ Department of Thoracic Surgery, Medical University of Lodz, Lodz, Poland
}

Videosurgery Miniinv 2014; 9 (3): 393-397

DOI: $10.5114 /$ wiitm.2014.44292

\begin{abstract}
Introduction: Progress in the development of surgical techniques has led to the growing use of video-assisted thoracoscopic surgery (VATS) techniques in mediastinal cyst treatment.

Aim: To present our experience of treating mediastinal cysts with the minimally invasive technique.

Material and methods: Fifty patients with mediastinal cysts were treated from 2001 to 2011. There were 32 women and 18 men. The age of the patients ranged from 17 to 72, the mean age being 42 years. All patients underwent basic preoperative diagnostic tests of the chest: X-ray, computed tomography (CT), bronchoscopy and spirometry; 4 patients underwent endobronchial ultrasound-guided transbronchial needle aspiration (EBUS-TBNA) and 3 fine needle aspiration biopsy (FNAB) of the described lesions.

Results: The VATS was performed in each case. Conversion was carried out due to superior mediastinal location in 10 cases and pleural adhesions in 3 cases. The partial resection of a cyst was performed in 3 patients. One patient was treated conservatively due to heart failure. In that patient the transthoracic needle aspiration of a cyst under ultrasound guidance using alcoholisation with $76 \%$ ethanol with a good effect was performed twice. Cyst recurrence was observed in 1 case.

Conclusions: The surgical access depends on the location of a cyst. The VATS resection of a superior mediastinal cyst is not always feasible. Surgery of mediastinal cysts is both diagnostic and curative.
\end{abstract}

Key words: video-assisted thoracoscopic surgery, mediastinal cyst, thoracotomy.

\section{Introduction}

Progress in the development of surgical techniques has led to the growing use of video-assisted thoracoscopic surgery (VATS) techniques as an alternative to classical surgical treatment methods. Currently VATS has become the standard in the treatment and diagnostics of many pulmonary diseases, e.g. pneumothorax, pleural diseases, circumferentially located pulmonary nodules, and some forms of lung cancer. Numerous data from the literature indicate that VATS techniques allow for better diagnostics and treatment of mediastinal diseases, including mediastinal cysts.

\section{Aim}

The aim of this paper is to present our experience of treating mediastinal cysts with the minimally invasive technique.

\section{Material and methods}

Fifty patients with mediastinal cysts were treated from 2001 to 2011. There were 32 women and 18 men. The age of the patients ranged from 17 to 72 , the mean age being 42 years. Most patients did not report clinical symptoms at the time of admission to hospital. Non-characteristic symptoms in the form of cough and chest pain were only observed. In

\section{Address for correspondence}

Mariusz P. Łochowski MD, Department of Thoracic Surgery, Medical University of Lodz, 62 Pabianicka St, 95-050 Lodz, Poland,

phone: +48 602776 887, e-mail: marilo@op.pl 
the group of asymptomatic patients the disease was most often detected incidentally during the radiological examination of the chest.

In all patients preoperative basic diagnostic tests of the chest were done, i.e. X-ray, computed tomography (CT), bronchoscopy and spirometry. Endobronchial ultrasound-guided transbronchial needle aspiration (EBUS-TBNA) was performed in 4 patients and fine needle aspiration biopsy (FNAB) of the described lesions in 3 patients. Surgical procedures were conducted mostly in the lateral position of the patient, under general anaesthesia, using a double lumen tube. We used a visual track with the optical system of $90^{\circ}$ and $30^{\circ}$. The first trocar, through which we introduced a camera, was usually placed in the midaxillary line in the $4^{\text {th }}-7^{\text {th }}$ intercostal spaces. Other trocars, 1 or 2 as required, were inserted using the triangle method. During the procedure 1 pleural drain was placed.

\section{Results}

Out of the 50 cases of mediastinal cysts, the lesion was located in the anterior mediastinum in 34 (Table I), in the superior mediastinum in 10 and in the posterior mediastinum in 6 patients. The choice of the surgical technique depended on the location of the lesion and its anatomical relations to important structures of the mediastinum. We began each operation with the VATS technique. The need to convert to mini-thoracotomy arose in the case of lesions located in the superior mediastinum (10 cas- es). Conversion due to pleural adhesions preventing preparation was performed in 3 cases. The partial resection of a cyst due to difficult surgical access was performed in 3 patients. The remaining wall of the cyst was destroyed using plasma-argon coagulation. We treated 1 patient conservatively due to heart failure (NYHA III/IV). In that patient, transthoracic needle aspiration of the cyst under ultrasound guidance using alcoholisation with $76 \%$ ethanol with a good effect was performed twice. The average time of hospitalisation in patients treated with the VATS technique was 4 days, whereas in patients treated applying the open technique it was 6 days. Cyst recurrence was observed in 1 case of a partially resected lesion situated within large vessels. In the other patients, no late complications were found.

\section{Discussion}

The term mediastinal cyst is used for well-demarcated round shadows covered with epithelium which contain fluid. They constitute about 15-20\% of all pathological masses of the mediastinum and may occur in all parts of the mediastinum. The most commonly encountered cysts of the mediastinum include: bronchial (Photo 1), pleuropericardial (Photo 2), enterogenic and thymic cysts (Photo 3 ). We can rarely find cystic forms of teratoma, lymphatic haemangioma, cysts of neurogenic origin, or hydatid cysts. Bronchial cysts most often occur in the anterior and superior mediastinum, pleuropericardial cysts in the anterior mediastinum, often in the right

Table I. Mediastinal cysts' characteristics

\begin{tabular}{|c|c|c|c|c|c|c|}
\hline Variables & \multicolumn{2}{|c|}{ Superior mediastinum } & \multicolumn{2}{|c|}{ Anterior mediastinum } & \multicolumn{2}{|c|}{ Posterior mediastinum } \\
\hline Number & \multicolumn{2}{|c|}{10} & \multicolumn{2}{|l|}{34} & \multicolumn{2}{|l|}{6} \\
\hline Female & \multicolumn{2}{|c|}{6} & \multicolumn{2}{|l|}{22} & \multicolumn{2}{|l|}{4} \\
\hline Male & \multicolumn{2}{|c|}{4} & \multicolumn{2}{|l|}{12} & \multicolumn{2}{|l|}{2} \\
\hline Surgical technique & \multicolumn{2}{|c|}{ VATS/thoracotomy } & \multicolumn{2}{|l|}{ VATS } & \multicolumn{2}{|c|}{ VATS } \\
\hline Conversion & \multicolumn{2}{|c|}{10} & \multicolumn{2}{|l|}{2} & \multicolumn{2}{|l|}{1} \\
\hline Recurrence & \multicolumn{2}{|c|}{-} & \multicolumn{2}{|l|}{1} & \multicolumn{2}{|l|}{-} \\
\hline Hist-pat. & $\begin{array}{l}\text { Bronchial c. } \\
\text { Thymic c. } \\
\text { Teratoma c. }\end{array}$ & $\begin{array}{l}4 \\
7 \\
1\end{array}$ & $\begin{array}{c}\text { Bronchial c. } \\
\text { Pleuropericardial c. }\end{array}$ & $\begin{array}{l}14 \\
13\end{array}$ & $\begin{array}{c}\text { Enterogenic c. } \\
\text { Bronchial c. }\end{array}$ & $\begin{array}{l}8 \\
3\end{array}$ \\
\hline $\begin{array}{l}\text { Average time } \\
\text { of hospitalisation [days] }\end{array}$ & \multicolumn{2}{|c|}{6} & \multicolumn{2}{|l|}{4} & \multicolumn{2}{|l|}{4} \\
\hline Deaths & \multicolumn{2}{|c|}{-} & \multicolumn{2}{|l|}{-} & \multicolumn{2}{|l|}{-} \\
\hline
\end{tabular}




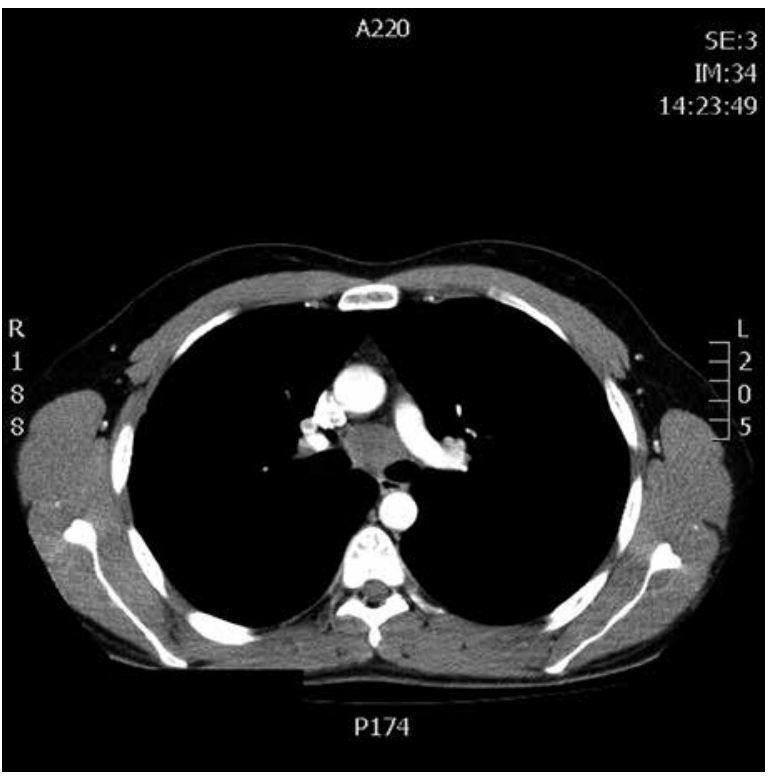

Photo 1. Example of a bronchial cyst located in the bifurcation of the trachea

heart-diaphragmatic angle, and enterogenic cysts in the posterior mediastinum [1, 2]. They may appear at different ages but most often in the fourth or fifth decades of life. They do not show predilection for gender, except for enterogenic cysts, which are most common in women [1]. Thymic cysts are most often found in the anterior and superior mediastinum. About $50 \%$ of congenital thymic cysts are detected in the first 2 decades of life, while acquired forms usually occur without symptoms in adult men. In the material presented by us the mean age of patients was 42 years, with the predominance of women.

The fact that most cysts were asymptomatic in the material collected by us is reflected in the literature. Other symptoms presented by patients are non-specific and occur secondarily to the disease. Such symptoms as cough or chest pain which can mimic a heart attack are connected with the location of the cyst and compression of adjacent structures. Other symptoms described in the literature are dyspnoea, fever, pneumonia, and haemoptysis [1-5]. Despite the standard diagnostic tests performed, it was impossible to make a diagnosis before treatment in most cases. Some characteristic features in the computed tomography of the chest, such as the location or content of fluid, in connection with clinical symptoms, may narrow the range of potential aetiologies. Even then we cannot clearly determine whether we are dealing with a cyst, abscess,

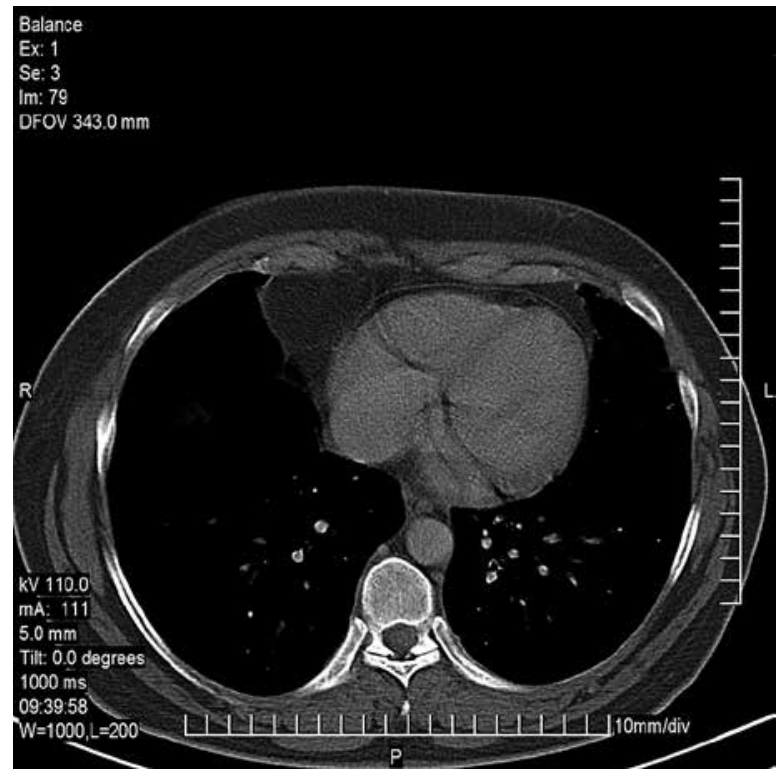

Photo 2. Pleuropericardial cyst

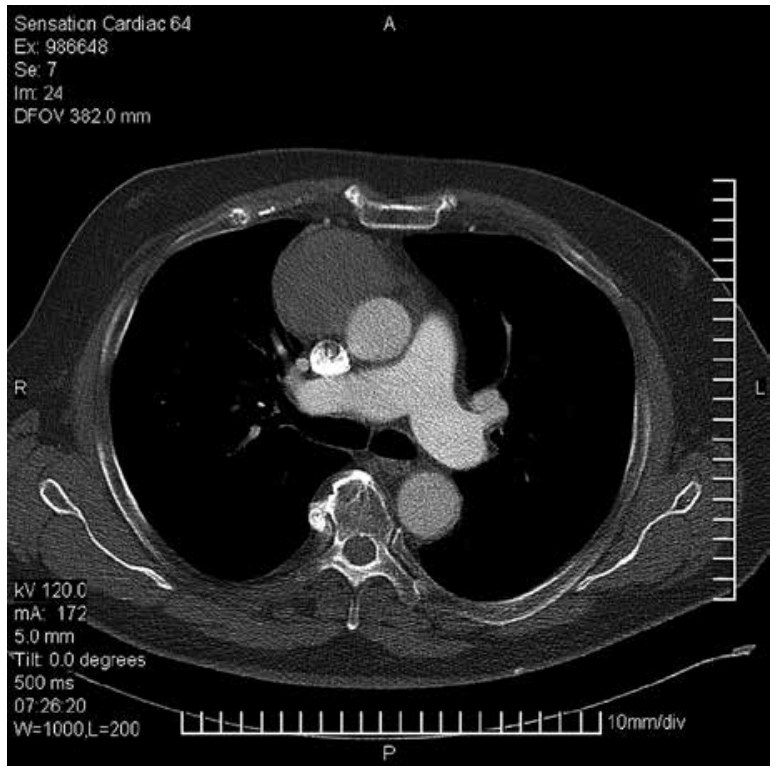

Photo 3. Thymic cyst located in the superior mediastinum

haemangioma or neurogenic or germinal tumour [6]. Therefore, medical treatment consisting in cyst resection was also diagnostic treatment, except for a few cases in which the diagnosis was made using FNAB or EBUS-TBNA. There are also rare cases of hydatid cysts, which should be excised completely as puncture poses a risk of anaphylactic shock and a fatal outcome [7]. Most mediastinal cysts were removed using the VATS method. Although the standards of conduct for the thoracoscopic removal of 
mediastinal cysts are still not precisely defined, some authors believe, as we do, that all mediastinal cysts may be subjected to the minimally invasive treatment of choice.

In our institution we began each operation with the VATS technique. An adhesion or invasion of important adjacent anatomical structures (e.g. the superior vena cava, oesophagus or trachea) resulted in conversion to mini-thoracotomy if the lesion was situated in the superior mediastinum (10 patients). In those cases complete removal was impossible so conversion was necessary to achieve radical resection. The choice of a proper surgical technique depends on cyst location. If VATS is impossible to perform, an alternative can be the open method. In all cases the cyst was visible through the thoracotomy site. Of course, if access through thoracotomy is difficult, full or partial sternotomy can also be performed. That access can be useful especially in cases of thymic cysts.

The main advantage of minimally invasive techniques is their milder perioperative trauma and discomfort for the patient, as we avoid cutting on a large length of the intercostal muscles and spreading the intercostals. The risk of breaking the ribs spread in the open method is also reduced to the minimum. That results in milder postsurgical pain and faster rehabilitation after the treatment, and thus a shorter stay in hospital [8]. In our case, the average duration of stay for patients subjected to VATS was 4 days (6 days for patients after thoracotomy). In patients who underwent thoracoscopy we can also observe a shorter duration of pleural drainage $[9,10]$. In our patients the drainage was on average removed during the first 2 days after the surgery.

The VATS technique involves optic system insertion into the pleural cavity and dividing the pleural layer over the cystic wall. Anterior mediastinal tumours are often in close proximity to the internal mammary artery, which is a common source of bleeding and reason for conversion. Bronchial or enteric cysts are sometimes communicating with the lumen of the trachea or bronchial tree. In such a situation the peduncle should be divided using endostaplers. Consequences of an injury are massive air leak or empyema requiring open surgery. In the case of neurenteric cysts, which often occur in the posterior mediastinum, the source of bleeding can be intercostal arteries. Access to a cyst can also be hampered by the descending aorta and the arch of the aorta on the left side. While managing the area of the aortopulmonary window or tracheo-oesophageal groove, the operator should be careful to avoid an injury of the left recurrent laryngeal nerve, which can lead to wheezing and vocal cord paralysis after surgery. In our material the main reason for conversion to thoracotomy was an upper mediastinal location (10 cases) and numerous pleural adhesions that prevented preparation (3 cases). Only severe adhesions are a contraindication to perform VATS. Other reasons for conversion described in the literature, apart from those listed, are bleeding, large size of the cyst, and poor exposure of the lesion [8-12]. In cases of more serious complications it is usually safer and necessary to convert to an open procedure. Due to possible complications some centres recommend referring patients to departments with experience of thoracic and minimally invasive surgery [12]. In the case of large cysts it was necessary to puncture and aspirate them. That treatment was usually provided at the end of the procedure, after the previous careful preparation of the cyst walls. In the case of accidental disruption of the continuity of the cyst, the content is immediately aspirated. The puncture procedure is sometimes performed prior to preparation to reveal anatomical relations more clearly and depends on the preferences of the centre where it is performed $[8,13]$. Recurrence is rare and mostly connected with the presence of the preserved epithelial lining of the cyst wall. We should always strive to completely excise the cyst with its content and, when that is impossible, we should strive to destroy the epithelium [8].

As already mentioned, cysts occur mostly in the fourth or fifth decade of life although they can be found in older patients. The authors are of the opinion that all detected cases of mediastinal cysts are an indication for surgery. However, older patients or those with many comorbidities which are contraindications to surgery, with moderately or mildly manifested symptoms of a mediastinal cyst, can be treated conservatively. However, if symptoms are life-threatening, surgery must always be considered. Treatment consisting in the transthoracic puncture of the cyst with simultaneous alcoholisation was applied in 1 patient disqualified from surgical treatment with a good result.

Post-hospital evaluation was performed in 45 patients. Patients were evaluated every 3 months during the first year, every 6 months in the second 
year and annually during the following period. The standard was to obtain an X-ray image during each visit and perform CT every 12 months. Cyst recurrence was observed in 1 case of a partially excised lesion after the first 3 months following surgery. This patient was observed during the next 3 years with no signs of further progression. In other patients no late complications were found. In the postoperative process there were no deaths and results of the thoracoscopic treatment of cysts were good.

\section{Conclusions}

Surgical access depends on the location of the cyst. VATS in the upper mediastinal cysts is not always feasible; an alternative is open procedures. Surgery of mediastinal cysts is both diagnostic and curative.

\section{References}

1. Zambudio AR, Lanzas JT, Calvo MJR, et al. Non-neoplastic mediastinal cysts. Eur J Cardiothorac Surg 2002; 22: 712-6.

2. Jeung MY, Gasser B, Gangi A, et al. Imaging of cystic masses of the mediastinum. RadioGraphics 2002; 22: S79-S93.

3. Koshy T, Sinha PK, Misra S, et al. Pericardial cyst. Ann Card Anaesth 2008; 11: 129-30.

4. Sarper A, Ayten A, Golbasi I, et al. Bronchogenic cyst. Tex Heart Inst J 2003; 30: 105-8.

5. Bouzas-Mosquera A, Alvarez-Garcia N, Peteiro J, et al. Pericardial cyst. Inter Med 2008; 47: 1819-20.

6. Quint L. Imaging of anterior mediastinal masses. Cancer Imaging 2007; 7: S56-62.

7. Traibi A, Atoini F, Zidane A, et al. Mediastinal hydatid cyst. J Chin Med Assoc 2010; 73: 3-7.

8. Giacomo T, Diso D, Anile M, et al. Thoracoscopic resection of mediastinal bronchogenic cysts in adults. Eur J Cardiothorac Surg 2009; 36: 357-9.

9. Tölg C, Abelin K, Laudenbach V, et al. Open vs thoracoscopic surgical management of bronchogenic cysts. Surg Endosc 2005; 19: 77-80.

10. Demmy TL, Krasna MJ, Detterbeck FC, et al. Multicenter VATS experience with mediastinal tumors. Ann Thorac Surg 1998; 66: 187-92.

11. Weber T, Roth TC, Beshay M, et al. Video-assisted thoracoscopic surgery of mediastinal bronchogenic cysts in adults: a single-center experience. Ann Thorac Surg 2004; 78: 987-91.

12. Neoral Č, Aujeský R, Škarda J, et al. Thoracoscopic treatment of benign esophageal tumors. Videosurgery Miniinv 2012; 7 : 294-8.

13. Gossot D, Izquierdo RR, Girard P, et al. Thoracoscopic resection of bulky intrathoracic benign lesions. Eur J Cardiothorac Surg 2007; 32: 848-51.

Received: 25.07.2013, accepted: 1.06.2014 